\title{
Larvicultura intensiva de sábalo (Prochilodus lineatus) con diferentes densidades de cría
}

\author{
Roux, J.P.; González, A.O.; Ortiz, J.; Sánchez, S.; Comolli, J. \\ Instituto de Ictiología del Nordeste, Facultad de Ciencias Veterinarias, Universidad Nacional del Nordeste \\ (UNNE), Sargento Cabral 2139, Corrientes (3400), Argentina. Tel.: (0379) 4425753. Int. 171. \\ E-mail: jproux@vet.unne.edu.ar
}

\begin{abstract}
Resumen
Roux, J.P.; González, A.O.; Ortiz, J.; Sánchez, S.; Comolli, J.: Larvicultura intensiva de sábalo (Prochilodus lineatus) con diferentes densidades de cría. Rev. vet. 26: 2, $143-$ 146, 2015. El objetivo del presente trabajo fue evaluar el efecto de la densidad de cría sobre la supervivencia y el peso final de larvas de Prochilodus lineatus cultivadas en sistema intensivo. Se evaluaron cuatro densidades de larvas de sábalo: 25, 50, 100 y 200 larvas/litro (T1, T2, T3 y T4, respectivamente). Se utilizaron 12 acuarios de pequeño volumen. Al final de la experiencia se procedió al recuento y pesaje de la totalidad de los peces, datos que se emplearon para estimar la tasa de supervivencia y el peso medio final, respectivamente. Para peso medio no se detectaron diferencias significativas entre tratamientos (T1: 36,53 mg; T2: 39,62 mg; T3: $37 \mathrm{mg}$ y T4 35,50 mg, $\mathrm{p} \geq 0,05$ ), mientras que en la tasa de supervivencia, T3 tuvo mejor rendimiento que T4 $(85,13 \%$ versus $68,13 \%, \mathrm{p} \leq 0,05)$, al tiempo que T1 y T2 presentaron valores intermedios y no se diferenciaron del resto. Bajo las condiciones de cría del presente trabajo, no se encontraron diferencias en el peso medio de las larvas a las densidades estudiadas.
\end{abstract}

Palabras clave: larvas de pez, Prochilodus lineatus, alimentación, artemia, densidad de cría, tasa de sobrevivencia.

\begin{abstract}
Roux, J.P.; González, A.O.; Ortiz, J.; Sánchez, S.; Comolli, J.: Intensive larviculture of sábalo (Prochilodus lineatus) at different breeding densities. Rev. vet. 26: 2, 143-146, 2015. The present work intends to evaluate the effect of stocking density on survival and final weight of Prochilodus lineatus larvae grown in an intensive system. Four densities of larvae were evaluated: 25, 50, 100 and 200 larvae/liter (T1, T2, T3 and T4, respectively). Twelve small volume tanks were used. At the end of the experiment all the fish were counted and weighed, data used to estimate the survival rate and the average final weight, respectively. For average weight, no significant differences were detected between treatments, while for the survival rate, T3 had better performance than T4. Groups T1 and T2 had intermediate values and did not differ from the rest. Under the rearing conditions of this study, no differences in larvae average weight were found for the considered densities.
\end{abstract}

Key words: fish larvae, Prochilodus lineatus, feeding, artemia, breeding density, survival rate.

\section{INTRODUCCIÓN}

El sábalo (Prochilodus lineatus) es una especie con una amplia distribución en Argentina, encontrándose en los ríos Paraná, Paraguay, Uruguay, Río de la Plata, Bermejo, Pilcomayo y tributarios ${ }^{19}$, siendo la especie de mayor importancia pesquera comercial de agua con-

Proyecto SGCYT (UNNE) B009/10

Presentado 34 Sesión Comunic. FCV-UNNE 2013

Recibido: 20 octubre 2014 / Aceptado: 18 de mayo 2015 tinental de la Argentina ${ }^{11}$. Presenta hábitos alimenticios iliófagos y está adaptado para consumir detritos orgánicos del fondo de ríos y lagunas $7,19,22$, cualidad que lo posiciona adecuadamente para la cría en policultivo, ya que permite aumentar la biomasa de peces de un estanque sin incrementar la oferta de alimento balanceado ${ }^{6,12}$. Esta especie podría incorporarse como acompañante en los cultivos de pacú (Piaractus mesopotamicus), bajo sistema semintensivo en estanques de tierra excavados ${ }^{14}$. 
El sábalo es un desovador total ${ }^{23}$, que presenta alta fecundidad y buena respuesta a la estimulación hormonal ${ }^{6}$, lo que permite planificar su producción a escala comercial, considerando la gran cantidad de huevos y larvas que pueden obtenerse por cada reproductor. En este contexto, la larvicultura aparece como una etapa crítica del ciclo productivo donde se debe garantizar la producción de una cantidad de individuos juveniles suficiente como para abastecer a los piscicultores de la región.

Larvas de diferentes especies de peces son criadas a densidades variables bajo condiciones controladas de laboratorio, que van desde de 10 a 300 larvas/litro, así como en estanques a cielo abierto ${ }^{2}$, donde las densidades van de 100 a 500 larvas $/ \mathrm{m}^{3}$. Si bien cada sistema tiene ventajas y desventajas, la larvicultura intensiva se generalizó ya que permite mejorar la tasa de supervivencia, y si bien suele presentar reducción del crecimiento, estrés y aparición de enfermedades ${ }^{3,15,16}$, permite contar con un elevado número de individuos al final de esta etapa.

Un factor que influye en la respuesta de las larvas de peces a la densidad de cría es la alimentación ${ }^{10}$; el uso de alimento vivo es necesario pero costoso e insuficiente a medida que las larvas crecen ${ }^{13,17}$, pero asegura altas tasas de supervivencia ${ }^{18}$.

En el presente trabajo se propuso evaluar el efecto de la densidad de cría sobre la supervivencia y el peso final de larvas de $P$. lineatus cultivadas en sistema intensivo de laboratorio.

\section{MATERIAL Y MÉTODOS}

La experiencia se llevó a cabo en las instalaciones experimentales del Instituto de Ictiología del Nordeste (INICNE), en la temporada reproductiva 2012-2013 y tuvo una duración de 28 días. Se utilizaron reproductores propios del instituto y se realizó la inducción hormonal con desove en seco e incubación en recipientes cónicos.

Fueron evaluadas cuatro densidades de larvas de sábalo: 25, 50, $100 \mathrm{y}$ 200 larvas/litro (grupos experimentales T1, T2, T3 y T4, respectivamente), con cuatro replicas cada uno. Para tal fin, se utilizaron 16 acuarios de 8 litros de capacidad y abastecidos con 5

litros de agua de perforación, con aireación continua y renovación a razón de 2 litros/hora. Doce lotes de larvas procedentes de un solo desove se alojaron en los acuarios a las densidades mencionadas al tercer día de vida (considerado el día 0 de la experiencia) cuando las mismas habían absorbido el saco vitelino.

Las larvas se alimentaron con nauplios de artemia utilizándose una ración consistente en 100 nauplios diarios por larva, la que fue subdividida y ofrecida en cuatro comidas al día durante la primera semana. Durante la segunda semana la ración se aumentó a 125 nauplios por día y fue ofrecida en tres oportunidades. En la tercera semana la ración diaria fue de 150 nauplios por día, en dos oportunidades. En la cuarta semana se estabilizó la ración en 200 nauplios diarios, ofrecidos en dos momentos.

Diariamente a las 9 y 16 horas se registraron parámetros de calidad del agua en los acuarios: $\mathrm{O}_{2}$ en $\%$ y $\mathrm{mg} /$ litro, temperatura del agua y temperatura ambiente máxima y mínima. Dos veces por semana se registraron valores de conductibilidad y $\mathrm{pH}$. Al final de la experiencia se procedió al recuento de la totalidad de los peces, así como al pesaje de cada lote, datos que se emplearon para estimar el peso medio final y la tasa de supervivencia.

Los resultados obtenidos se evaluaron mediante ANOVA a una vía para cada una de las variables analizadas. Cuando se detectaron diferencias significativas $(p<0,05)$, se analizaron las tendencias polinómicas de la densidad de cría considerando los efectos lineal, cuadrático y cúbico. Todas las pruebas estadísticas se realizaron con el programa Infostat versión $2012^{9}$.

\section{RESULTADOS Y DISCUSIÓN}

Las variables obtenidas de calidad de agua se encontraron dentro del rango adecuado para la cría de la especie; solamente se pudo observar una acidificación del agua con un rango de valores entre 5,85 y 6,87 (Tabla 1).

Las variaciones normales de $\mathrm{pH}$ para peces de agua dulce varían entre 6 y $9^{4}$. En trabajos con alevines de $R$. quelen se comprobó que variaciones entre 4 y 9,5 no afectan la tasa de supervivencia tras 4 días de exposición ${ }^{25,26}$. En ambientes naturales los alevines de $P$. lineatus se desarrollan normalmente con $\mathrm{pH}$ que variaron entre 5,1 y 9,1 ${ }^{24}$. En producción, el sábalo tolera

Tabla 1. Calidad de agua en acuarios de larvicultura de sábalo ( $P$. lineatus).

\begin{tabular}{cccccccccc}
\hline \multirow{2}{*}{ tttrat. } & \multicolumn{5}{c}{$9 \mathrm{~h}$} & \multicolumn{5}{c}{$16 \mathrm{~h}$} & \multirow{2}{*}{\begin{tabular}{c} 
cond. \\
\cline { 2 - 6 }
\end{tabular}} & $\mathrm{T}^{\mathrm{o}}\left({ }^{\circ} \mathrm{C}\right)$ & $\mathrm{O}_{2}(\%)$ & $\mathrm{O}_{2}(\mathrm{mg} / \mathrm{l})$ & $\mathrm{T}^{\mathrm{o}}\left({ }^{\circ} \mathrm{C}\right)$ & $\mathrm{O}_{2}(\%)$ & $\mathrm{O}_{2}(\mathrm{mg} / \mathrm{l})$ & & $(\mu \mathrm{cm})$ \\
\hline 1 & 24,8 & 74,9 & 6,2 & 25,4 & 77,5 & 6,3 & & 6,2 & 89,2 \\
2 & 24,7 & 72,3 & 6,0 & 25,7 & 76,1 & 6,3 & & 6,2 & 89,0 \\
3 & 24,8 & 68,9 & 5,7 & 25,8 & 73,6 & 6,0 & 6,2 & 89,7 \\
4 & 25,1 & 55,4 & 4,7 & 26,0 & 54,8 & 4,5 & 6,2 & 92,0 \\
\hline
\end{tabular}

trat.: tratamiento; $\mathrm{T}^{\mathrm{o}}$ : temperatura del agua; $\mathrm{O}_{2}(\%)$ : concentración de oxígeno; cond.: conductividad del agua.

Tabla 2. Tasa de supervivencia y peso individual de alevines de sábalo (P.lineatus) criados en acuarios $(\overline{\mathrm{x}} \pm \mathrm{DE})$.

\begin{tabular}{ccc}
\hline TR (larvas/L) & TS $(\%)$ & PI $(\mathrm{mg})$ \\
\hline T1 25 & $81,80 \pm 5,75 \mathrm{ab}$ & $36,5 \pm 3,51 \mathrm{a}$ \\
T2 50 & $82,10 \pm 9,11 \mathrm{ab}$ & $39,6 \pm 4,16 \mathrm{a}$ \\
T3 100 & $85,13 \pm 7,23 \mathrm{~b}$ & $37,1 \pm 2,80 \mathrm{a}$ \\
T4 200 & $68,13 \pm 10,22 \mathrm{a}$ & $35,6 \pm 3,44 \mathrm{a}$ \\
\hline
\end{tabular}

TR: tratamiento, TS: tasa de supervivencia, PI: peso individual. Letras diferentes indican diferencias significativas entre tratamientos $(p<0,05)$. 


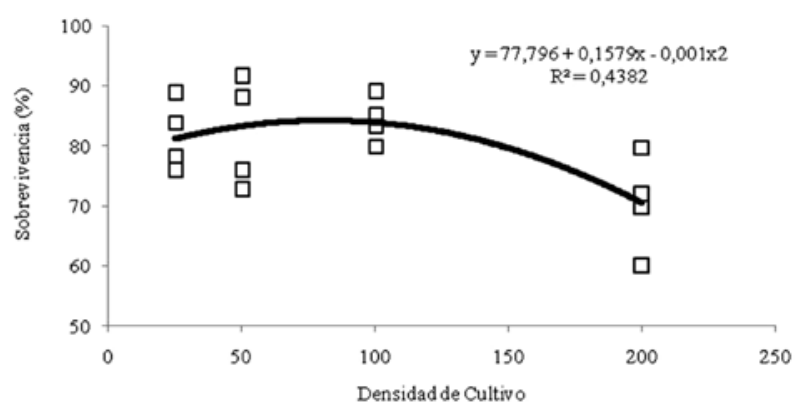

Figura 1. Relación entre supervivencia y densidad de cultivo (larvas/litro).

variaciones de 4,08 a 9,72 sin presentar mortalidad, al menos por 5 días ${ }^{27}$. Los valores del ensayo no se encuentran entre los más aptos para la cría de esta especie pero dado el margen de tolerancia, no habría influencia de este parámetro en el resultado final.

Los promedios estimados para las variables tasa de supervivencia y peso medio final se presentan en la Tabla 2. El análisis estadístico no detectó diferencias significativas entre tratamientos para el peso medio final ( $p \geq 0,05$ ), mientras que en la tasa de supervivencia, se estimó un efecto cuadrático $(\mathrm{p}<0,05)$. En esta variable puede apreciarse que la supervivencia presentaría su máximo valor alrededor de las 100 larvas/litro, descendiendo hacia las densidades mayores (Figura 1).

Las densidades sugeridas para la larvicultura intensiva varían según la especie. Para Pseudoplatystoma corruscans la densidad de 15 larvas/litro arrojó mejor supervivencia que valores más altos, cercanos a 45 larvas/litro ${ }^{5}$, sin relación entre densidad de cría y crecimiento. Para Rhamdia sebae las densidades de 25 larvas/litro obtuvieron mejores tasas de supervivencia y ganancias de peso que las densidades mayores ${ }^{8}$.

En el caso de Steindachneridion scriptum, se obtuvieron buenos resultados a densidades de 10 larvas/ litro ${ }^{20}$. La cría de poslarvas de salmón de río (Brycon orbignyanus) presentó mejores tasas de supervivencia a densidades de 10 larvas/litro, y al igual que el presente trabajo, no se observaron variaciones en el crecimiento y peso final comparado con otras densidades (20 y 30 larvas/litro) ${ }^{21}$.

Para Prochilodus magdalenae se sugirieron densidades de 10 larvas/litro con tasa de supervivencia de $73,2 \%$; tal porcentaje fue inferior al del presente trabajo con similares condiciones de cultivo. Otra posibilidad para esta especie consiste en utilizar densidades de 100 a 200 larvas /litro ${ }^{1}$ en sistema de alimentación temprana para alevines hasta los tres días en estanques de alevinaje, los valores propuestos se encuentran dentro de los verificados en el presente ensayo.

Se concluye que la utilización de densidades próximas a 100 larvas/litro permiten obtener la misma supervivencia y pesos finales que las logradas con densidades menores, dado que no hubo diferencias significativas en el peso final de las larvas estudiadas.

\section{REFERENCIAS}

1. Atencio V. 2001. Producción de alevinos de especies nativas. Rev MVZ Córdoba 6: 9-14.

2. Atencio V, Kerguelén E, Wadnipar L, Narváez A. 2003. Manejo de la primera alimentación del bocachico (Prochilodus magdalenae). Rev MVZ Córdoba 8: 254-260.

3. Barcellos LJ, Souza SD, Woehl VM. 2000. Estresse em peixes: fisiologia da resposta ao estresse, causas e conseqüências. Boletim Instit Pesca 26: 99-111.

4. Boyd CE. 1998. Water quality management for pond aquaculture: research and development. Int Center Aquacult \& Aquatic Environ 43: 1-37.

5. Campagnolo R, Nuñer ADO. 2006. Sobrevivência e crescimento de larvas de surubim, Pseudoplatystoma corruscans (Pisces, Pimelodidae), em diferentes densidades de estocagem. Acta Sci Anim Sc 28: 231-237.

6. Campos J. 2010. Género Prochilodus (sábalo). En: Peces nativos de agua dulce de América del Sur de interés para la acuicultura (Flores Nava A, Brown A, Ed), Serie Acuicultura en Latinoamérica $\mathrm{N}^{\circ}$ 1, FAO, Santiago (Chile), $200 \mathrm{p}$.

7. Casciotta J, Almirón A, Bechara J. 2005. Peces del Iberá, hábitat y diversidad, Ed. Fundación Ecos, La Plata (Argentina), $244 \mathrm{p}$.

8. David Ruales CA, Castañeda GD, Cruz CI, Lenis GA, Castro JD, Álvarez MA, Martínez ES. 2011. Efecto de la densidad de siembra sobre la larvicultura de guabina (Rhamdia sebae cf) en sistema de recirculación. http://repository.lasallista.edu .co/dspace/bitstream/10567/62/1/189-198.pdf

9. Di Rienzo JA, Casanoves F, Balzarini MG, González L, Tablada M, Robledo CW. InfoStat versión 2012, FCA (Córdoba, Argentina), http://www.infostat.com.ar

10. Dou S, Masuda R, Tanaka M, Tsukamoto K. 2003. Identification of factors affecting the growth and survival of the settling Japanese flounder larvae, Paralichthys olivaceus. Aquaculture 218: 309-327.

11. Flores A, Münzemayer C. 2010. Diagnóstico de oportunidades de transferencia de tecnología en acuicultura y pesca continental en países de América del Sur. Serie Acuicultura en Latinoamérica No 2, FAO, Santiago (Chile), 67 p.

12. Graeff A, Tomaselli A. 2011. Policultivo de carpas com introdução crescente do Curimatã (Prochilodus scropha) como espécie principal. Rev Electrón Vet 12: 10.

13. Hernández D, Domitrovic H, Sánchez E. 2006. Evaluación de diferentes dietas en la primera alimentación del bagre sudamericano (Rhamdia quelen). Anales IV Congr Iberoam Virtual Acuicul, Zaragoza (España), p. 1151-1155.

14. Hepher B, Pruginin Y. 1985. Cultivo de peces comerciales, Limusa (México), $316 \mathrm{p}$.

15. Jobling M. 1994. Fish bioenergetics, Chapman \& Hall, London, $294 \mathrm{p}$.

16. King NJ, Howell WH, Huber M, Bengtson DA. 2000. Effects of larval stocking density on laboratory-scale and commercial-scale production of summer flounder Paraliehthys dentatus. J World Aquacult Soc 31: 436-445.

17. Kolkovski S. 2001. Digestive enzymes in fish larvae and juveniles. Implications and applications to formulated diets. Aquaculture 200: 181-201. 
18. Luchini L, Avendaño T. 1985. Primer alevinaje de bagre sudamericano, Rhamdia sapo en condiciones controladas. Rev Asoc Cien Nat Litoral (Santa Fe, Argentina) 16: 137147.

19. Ringuelet R, Aramburu R, Alonso A. 1967. Los peces argentinos de agua dulce, Ed. Comisión de Investigación Científica, La Plata, Argentina, 602 p.

20. Schütz JH, Weingartner M, Zaniboni FE, Nuñer AP. 2008. Crescimento e sobrevivência de larvas de suruvi Steindachneridion scriptum nos primeiros dias de vida: influência de diferentes alimentos e fotoperíodos. Boletim Instit Pesca 34: 443-451.

21. Silva MO, Logato PV, Murgas LD, Ribeiro PA, Maria AN. 2009. Crecimiento y supervivencia de postlarvas de piracanjuba (Brycon orbignyanus). Arch Zoot 58: 285-288.

22. Sverlij S, Espinach A, Orti G. 1993. Sinopsis de los datos biológicos y pesqueros del sábalo Prochilodus lineatus (Valenciennes, 1847), Publ. FAO, Roma, 64 p.

23. Telichevsky S, Fortuny A, Picotti G. 1987. Biología reproductiva del sábalo Prochilodus lineatus Holmberg del Río Paraná en el área de Bella Vista. Informe del Instituto Nacional de Investigación y Desarrollo Pesquero (Convenio AyE-INIDEP), Buenos Aires, $17 \mathrm{p}$.
24. Thomaz SM, Roberto MD, Bini LM, Vazzoler AE, Agostinho AA. 1997. Caracterização limnológica dos ambientes aquáticos e influência dos níveis fluviométricos. In: A planície de inundação do alto rio Paraná: aspectos físicos, biológicos e socioeconômicos (Vazzoler AE, Agostinho AA, Hahn NS, Ed), Eduem, Maringá (Brasil) p. 73-102.

25. Townsend C, Baldisserotto B. 2001. Survival of silver catfish fingerlings exposed to acute changes of water $\mathrm{pH}$ and hardness. Aquacult Int 9: 413-419.

26. Zaions M, Baldisserotto B. $2000 . \mathrm{Na}^{+}$and $\mathrm{K}^{+}$body levels and survival of fingerlings of Rhamdia quelen (Siluriformes, Pimelodidae) exposed to acute changes of water pH. Ciênc Rural 30: 1041-1045

27. Zaniboni E, Meurer S, Golombieski J, Silva L, Baldisserotto B. 2002. Survival of Prochilodus lineatus (Valenciennes) fingerlings exposed to acute $\mathrm{pH}$ changes. Acta Scientiarum Anim Sci 24: 917-920.

\section{Asociación Cooperadora de la Facultad de Ciencias Veterinarias Universidad Nacional del Nordeste}

Personería Jurídica No 647/92 y 912/00

Sargento Cabral 2139

3400 Corrientes

Argentina

La Asociación Cooperadora de la Facultad de Ciencias Veterinarias de la UNNE fue constituida el 10 de diciembre del año 1991 como entidad de bien público, con el objeto de promover y coadyuvar las actividades científicas, educativas y culturales relacionadas con las Ciencias Veterinarias. En tal sentido, implementa acciones para colaborar con la enseñanza, extensión, actualización y difusión científica que realiza dicha casa de estudios.

\section{Beneficios que brinda a sus asociados:}

- Fotocopias con descuentos especiales en la Fotocopiadora Copias.com que funciona dentro del predio de la Facultad de Ciencias Veterinarias.

- Descuentos para la adquisición de libros de distintas editoriales.

- Descuentos especiales en otros rubros.

Tel. (0379) 4425753 interno 186•Página Web www.vet.unne.edu.ar•E-mail: cooperadora@vet.unne.edu.ar 